\title{
Association between Helicobacter pylori Infection and Risk of Osteoporosis in Elderly Taiwanese Women with Upper Gastrointestinal Diseases: A Retrospective Patient Record Review
}

\author{
Shih-Chun Lin, ${ }^{1}$ Malcolm Koo, ${ }^{2,3}$ and Kun-Wei Tsai ${ }^{1}$ \\ ${ }^{1}$ Department of Geriatrics, Dalin Tzu Chi Hospital, Buddhist Tzu Chi Medical Foundation, 2 Minsheng Road, Dalin, \\ Chiayi 62247, Taiwan \\ ${ }^{2}$ Department of Medical Research, Dalin Tzu Chi Hospital, Buddhist Tzu Chi Medical Foundation, 2 Minsheng Road, Dalin, \\ Chiayi 62247, Taiwan \\ ${ }^{3}$ Dalla Lana School of Public Health, University of Toronto, 155 College Street, 6th Floor, Toronto, ON, Canada M5T 3M7
}

Correspondence should be addressed to Malcolm Koo; m.koo@utoronto.ca and Kun-Wei Tsai; cktsai@aol.com

Received 21 February 2014; Revised 29 April 2014; Accepted 30 April 2014; Published 13 May 2014

Academic Editor: Vikram Kate

Copyright (C) 2014 Shih-Chun Lin et al. This is an open access article distributed under the Creative Commons Attribution License, which permits unrestricted use, distribution, and reproduction in any medium, provided the original work is properly cited.

\begin{abstract}
Introduction. Helicobacter pylori (H. pylori) infection could lead to chronic local and systemic immune response. The resulting increase in proinflammatory cytokines could affect bone resorption and might increase the risk of osteoporosis. This study aimed to investigate the association between $H$. pylori infection and osteoporosis in elderly female patients with upper gastrointestinal diseases. Methods. A retrospective patient record review study was conducted in a regional teaching hospital in south Taiwan. Relevant information on female patients aged 65 and over who were diagnosed with diseases of esophagus, gastric ulcer, or duodenal ulcer during January 2008 to December 2010 were abstracted. Association between H. pylori infection and osteoporosis was evaluated using multivariate logistic regression analysis. Results. Of the 365 patients with a mean age of 77.3 years, 77 (21.1\%) had $H$. pylori infection and 101 (27.7\%) had been diagnosed with osteoporosis. Multivariate logistic regression analysis revealed that osteoporosis was significantly associated with $H$. pylori infection (adjusted odds ratio $=2.03,95 \%$ confidence interval $=1.14-$ 3.62) after adjusting for age group, body mass index group, and use of proton pump inhibitor. Conclusion. Osteoporosis was found to be associated with $H$. pylori infection in Taiwanese female patients with upper gastrointestinal diseases. Further studies with information on potential confounders are needed to confirm the association.
\end{abstract}

\section{Background}

Osteoporosis is a major public health problem affecting up to one in three women [1]. It has been estimated that approximately half of the hip fractures worldwide are a result of osteoporosis [2]. The morbidity, disability, and mortality associated with osteoporosis can impose a substantial burden on affected individuals, their families, and the health care system [3]. Therefore, identifying risk factors and at risk populations for osteoporosis are important for the prevention of hip fracture.

Helicobacter pylori (H. pylori) is a spiral-shaped, Gramnegative bacterium that colonizes the gastric mucosa of more than $50 \%$ of human population. It can elicit lifelong inflammatory and immune responses in infected individuals. It is strongly associated with chronic gastritis, peptic ulcer, gastric mucosa-associated lymphoid tissue (MALT) lymphoma, and gastric cancer [4]. In addition, H. pylori infection has previously been suggested to be involved in the pathogenesis of a number of extradigestive disorders including cardiovascular, skin, rheumatic, liver cirrhosis, diabetes mellitus, and respiratory diseases [5].

$H$. pylori infection can increase the systemic levels of inflammatory cytokines and bone turnover regulating cytokines such as tumor necrosis factor-alpha, interleukin-1, and interleukin-6 [6]. Since these factors are involved in 
the pathogenesis of osteoporosis, it is plausible that individuals with $H$. pylori infection are at an increased risk of osteoporosis. However, few studies have investigated this association except in a study of 80 osteoporotic male patients and 160 controls where the results showed that individuals infected by CagA-positive $H$. pylori strains was more prevalent in men with osteoporosis [7]. On the other hand, $H$. pylori infection was not accompanied by significant changes in biochemical markers of bone metabolism in a study of $41 \mathrm{H}$. pylori positive children [8]. In addition, to our knowledge, only one study has specifically evaluated the association in elderly females [9]. Therefore, using retrospective patient medical record analysis, we investigated the association between $H$. pylori infection and osteoporosis among elderly Taiwanese women with upper gastrointestinal diseases.

\section{Methods}

2.1. Study Design and Data Collection. A retrospective patient medical record analysis was performed using the data on the computerized patients' record database in a regional hospital in south Taiwan. The study protocol was approved by the institutional review board of the Dalin Tzu Chi Hospital, Buddhist Tzu Chi Medical Foundation (no. B10003001). Informed consent was not required because of the nature of the study. Female patients were included in the study if they were 65 years or older and were diagnosed with diseases of esophagus, gastric ulcer, or duodenal ulcer (International Classification of Diseases, Ninth Revision, Clinical Modification [ICD-9-CM] codes of 530.xx to 532.xx) between January 1, 2008, and December 31, 2010.

A medical record abstraction form was used to collect information on age, body weight, height, smoking, alcohol use, $H$. pylori testing, diagnosis of osteoporosis, the use of proton pump inhibitor (PPI), and antiosteoporotic medications. The presence of $H$. pylori infection was determined by histological examination [10], rapid urease test [11], or both. Body mass index (BMI) was calculated as the weight in kilograms divided by the height in meters squared. It was further divided into four categories according to the classification of the Department of Health of Taiwan: underweight $\left(B M I<18.5 \mathrm{~kg} / \mathrm{m}^{2}\right)$, normal weight $(\mathrm{BMI}=18.5-$ $\left.23.9 \mathrm{~kg} / \mathrm{m}^{2}\right)$, overweight $\left(\mathrm{BMI}=24.0-26.9 \mathrm{~kg} / \mathrm{m}^{2}\right)$, and obese $\left(\mathrm{BMI} \geq 27 \mathrm{~kg} / \mathrm{m}^{2}\right)$. Osteoporosis was defined by either dual energy X-ray absorptiometry (DXA) scan [12] or use of osteoporosis medications. The latter was mainly as a result of bony fractures related to osteoporosis rather than use for primary prevention of osteoporosis.

2.2. Statistical Analyses. Categorical variables were expressed as frequencies and percentages and Fisher's exact test was used for their comparisons. Multiple logistic regression analyses were used to evaluate the association between osteoporosis and $H$. pylori infection, adjusting for age, BMI, and the use of proton pump inhibitor. Hosmer-Lemeshow statistics were used to assess model fit. A two-tailed $P$ value $<0.05$ was considered statistically significant.

\section{Results}

A total of 671 female patients with upper gastrointestinal diseases were identified from the medical records. Of them, $494(73.6 \%)$ had undergone testing for $H$. pylori and a further 129 patients were excluded from the final analysis due to missing data in other biochemical variables. The mean age of these 365 patients was 77.3 years (median $=77$, range $=65-97$ years). Of them, 77 (21.1\%) were tested positive for $H$. pylori and $101(27.7 \%)$ had been diagnosed with osteoporosis.

Table 1 compares the characteristics between patients tested positive and negative for $H$. pylori. The group tested negative for $H$. pylori was younger $(P=0.061)$ and had a higher proportion with gastric ulcer $(P=0.051)$. The group tested positive for $H$. pylori had significantly higher proportion of duodenal ulcer $(P=0.033)$. No significant differences were observed in the distributions of tertiles of PPI use between the two groups. Results from multivariate logistic regression analyses revealed that osteoporosis was significantly associated with $H$. pylori infection (adjusted odds ratio $=2.03,95 \%$ confidence interval $=1.14-3.62)$ after adjusting for age group, body mass index group, and use of proton pump inhibitor (Table 2).

\section{Discussion}

The key finding of retrospective patient medical record analysis was that $H$. pylori infection was significantly associated with osteoporosis in elderly Taiwanese women with upper gastrointestinal diseases. The odds of osteoporosis in patients tested positive for $H$. pylori infection were twice of that tested negative and the association was independent of age, BMI, and PPI use.

To date, there was only one cross-sectional study designed to specifically evaluate the association between $H$. pylori infection and osteoporosis in postmenopausal women [9]. The authors of the study concluded that $H$. pylori infection was not significantly different between patients with or without osteoporosis $(P=0.22)$. Nevertheless, with only 50 subjects, the study suffered from a low statistical power to detect the association when it truly exists (power $=33 \%$ ). Studies with adequate sample sizes are needed to ensure that a reasonable statistical power can be achieved.

Although the exact mechanisms underlying the observed association between $H$. pylori infection and osteoporosis are not clear, several possible explanations have previously been postulated. First, chronic infection of $H$. pylori can increase the release of cytokines, such as tumor necrosis factor(TNT-) $\alpha$, interleukin-1, and interleukin-6. It has been demonstrated that increased levels of interleukin-1 and TNF$\alpha$ can lead to bone resorption [13] and, therefore, may increase the risk of osteoporosis.

In addition, $H$. pylori infection has shown to decrease the levels of vitamin $B_{12}[8,14]$. Low levels of vitamin 
TABLE 1: Characteristics of patients according to $H$. pylori infection.

\begin{tabular}{|c|c|c|c|c|}
\hline \multirow{3}{*}{ Variable } & \multicolumn{3}{|c|}{ Frequency (\%) } & \multirow{3}{*}{$P$} \\
\hline & Total & H. pylori positive & H. pylori negative & \\
\hline & $365(100)$ & $77\left(21.1^{\mathrm{a}}\right)$ & $288(78.9)$ & \\
\hline Age (years) & & & & 0.061 \\
\hline $65-74$ & $145\left(39.7^{\mathrm{b}}\right)$ & $22(28.6)$ & $123(42.7)$ & \\
\hline $75-84$ & $150(41.1)$ & $36(46.8)$ & $114(39.6)$ & \\
\hline$\geq 85$ & $70(19.2)$ & $19(24.7)$ & $51(17.7)$ & \\
\hline Body mass index ${ }^{c}$ & & & & 0.938 \\
\hline Underweight & 39 (11.7) & $9(13.2)$ & $30(11.4)$ & \\
\hline Normal & $153(46.1)$ & $30(44.1)$ & $123(44.1)$ & \\
\hline Overweight & $73(22.0)$ & $16(23.5)$ & $57(23.5)$ & \\
\hline Obese & $67(20.2)$ & $13(19.1)$ & $54(19.1)$ & \\
\hline Smoking & & & & 0.742 \\
\hline Yes & $13(3.6)$ & $3(3.9)$ & $10(3.5)$ & \\
\hline No & $351(96.4)$ & $74(96.1)$ & $277(96.5)$ & \\
\hline Alcohol use & & & & $>0.999$ \\
\hline Yes & $6(1.6)$ & $1(1.3)$ & $5(1.7)$ & \\
\hline No & $358(98.4)$ & $76(98.7)$ & $282(98.3)$ & \\
\hline Diseases of esophagus (ICD-9-CM ${ }^{\mathrm{d}}$ code 530 ) & & & & 0.695 \\
\hline Yes & $144(39.5)$ & $32(41.6)$ & $112(38.9)$ & \\
\hline No & $221(60.5)$ & $45(58.4)$ & $176(61.1)$ & \\
\hline Gastric ulcer (ICD-9-CM code 531) & & & & 0.051 \\
\hline Yes & $216(59.2)$ & $38(49.4)$ & $178(61.8)$ & \\
\hline No & $149(40.8)$ & $39(50.6)$ & $110(38.2)$ & \\
\hline Duodenal ulcer (ICD-9-CM code 532) & & & & 0.033 \\
\hline Yes & $56(15.3)$ & $18(23.4)$ & $38(13.2)$ & \\
\hline No & $309(84.7)$ & $59(76.6)$ & $250(86.8)$ & \\
\hline Proton pump inhibitor use & & & & 0.289 \\
\hline Not use & $23(6.3)$ & $2(2.6)$ & $21(7.3)$ & \\
\hline$<2$ month & $115(31.5)$ & $23(29.9)$ & $92(31.9)$ & \\
\hline$\geq 2$ months & $227(62.2)$ & $52(67.5)$ & $175(60.8)$ & \\
\hline
\end{tabular}

${ }^{a}$ Row percentage.

${ }^{\mathrm{b}}$ Column percentage.

${ }^{c} 33$ missing values in body mass index, 1 missing value in smoking and alcohol use.

${ }^{\mathrm{d}}$ International Classification of Diseases (ICD), Ninth Revision (ICD-9), Clinical Modification.

$\mathrm{B}_{12}$ are considered as a risk factor for osteoporosis [15, 16]. Furthermore, medications for the treatment of upper gastrointestinal disorders such as PPI might affect calcium absorption and thereby increase the risk of osteoporosis. A prospective cohort study conducted over a period of 10 years in Canada reported that PPI use was associated with significantly lower baseline body mineral density at the femoral neck and total hip. However, PPI use over 10 years did not appear to be associated with accelerated loss of bone mineral density [17]. A review study on 14 observational studies published from 1980 to early 2011 concluded that PPI use was associated with a modest increase in the risk of hip fracture and vertebral fracture. Nevertheless, some studies showed conflicting results, possibly as a result of residual confounding [18]. In our study of patients with upper gastrointestinal diseases, PPI use was not significantly associated with osteoporosis. A possible reason is that longterm use of PPI is not under the coverage of the Taiwan National Health Insurance scheme and, therefore, patients generally use it for only 2 to 4 months. PPI use was also not significantly different between $H$. pylori positives and negatives for the same reason of lack of long-term insurance coverage.

Findings from this study should be weighed within the confines of the limitations of our data source. First, the study subjects came from a single regional hospital, which might not be representative of other settings. Second, retrospective studies based on abstraction of medical records are constrained by the accuracy and completeness of the records. Third, not all patients with upper gastrointestinal diseases were tested for presence of $H$. pylori. Therefore, we could not rule out the presence of selection bias. Fourth, 
TABLE 2: Multivariate logistic regression analysis of osteoporosis $(N=332)$.

\begin{tabular}{|c|c|c|c|}
\hline Variable & $n(\%)$ & Odds ratio $(95 \% \mathrm{CI})$ & $P$ \\
\hline \multicolumn{4}{|l|}{ Age (years) } \\
\hline $65-74$ & $138(41.6)$ & 1.00 & \\
\hline $75-84$ & $132(39.8)$ & $1.06(0.62-1.82)$ & 0.838 \\
\hline$\geq 85$ & $62(18.7)$ & $0.93(0.45-1.90)$ & 0.830 \\
\hline \multicolumn{4}{|c|}{ Body mass index group } \\
\hline Normal & $153(46.1)$ & 1.00 & \\
\hline Underweight & $39(11.8)$ & $0.62(0.27-1.43)$ & 0.264 \\
\hline Overweight & $73(22.0)$ & $0.49(0.24-0.96)$ & 0.039 \\
\hline Obese & $67(20.2)$ & $0.95(0.51-1.79)$ & 0.881 \\
\hline \multicolumn{4}{|c|}{ Proton pump inhibitor use } \\
\hline Not use & $23(6.9)$ & 1.00 & \\
\hline$<2$ month & $101(30.4)$ & $1.33(0.44-4.02)$ & 0.610 \\
\hline$\geq 2$ months & $208(62.7)$ & $1.32(0.46-3.79)$ & 0.606 \\
\hline \multicolumn{4}{|l|}{ H. pylori } \\
\hline Negative & $264(79.5)$ & 1.00 & \\
\hline Positive & $68(20.5)$ & $2.03(1.14-3.62)$ & 0.016 \\
\hline
\end{tabular}

Since there were no drinkers or smokers in the patients with osteoporosis in our study, their associations with osteoporosis were not analyzed. Only 332 subjects were included in the final model due to 33 missing values in body mass index.

measurement of systemic inflammatory markers was not available to confirm whether they were elevated in patients with $H$. pylori infection.

\section{Conclusions}

Results from our retrospective patient medical record analysis indicated that $H$. pylori infection was associated with osteoporosis in elderly female Taiwanese. Further studies using prospective design with information on systemic inflammatory markers and potential confounding factors are needed to confirm the association.

\section{Abbreviations}

BMI: $\quad$ Body mass index

H. pylori: Helicobacter pylori

PPI: $\quad$ Proton pump inhibitor.

\section{Conflict of Interests}

The authors declare that there is no conflict of interests regarding the publication of this paper.

\section{Acknowledgment}

This work was supported by grants from the Dalin Tzu Chi Hospital, Buddhist Tzu Chi Medical Foundation (no. B10003001).

\section{References}

[1] R. W. Keen, "Burden of osteoporosis and fractures," Current Osteoporosis Report, vol. 1, no. 2, pp. 66-70, 2003.
[2] A. Odén, E. V. McCloskey, H. Johansson, and J. A. Kanis, "Assessing the impact of osteoporosis on the burden of hip fractures," Calcified Tissue International, vol. 92, no. 1, pp. 4249, 2013.

[3] D. J. Becker, M. L. Kilgore, and M. A. Morrisey, "The societal burden of osteoporosis," Current Rheumatology Reports, vol. 12, no. 3, pp. 186-191, 2010.

[4] P. Ruggiero, "Helicobacter pylori and inflammation," Current Pharmaceutical Design, vol. 16, no. 38, pp. 4225-4236, 2010.

[5] K. X. Papamichael, G. Papaioannou, H. Karga, A. Roussos, and G. J. Mantzaris, "Helicobacter pylori infection and endocrine disorders: is there a link?" World Journal of Gastroenterology, vol. 15, no. 22, pp. 2701-2707, 2009.

[6] L. G. Raisz, "Physiology and pathophysiology of bone remodeling," Clinical Chemistry, vol. 45, no. 8, part 2, pp. 1353-1358, 1999.

[7] N. Figura, L. Gennari, D. Merlotti et al., "Prevalence of Helicobacter pylori infection in male patients with osteoporosis and controls," Digestive Diseases and Sciences, vol. 50, no. 5, pp. 847852, 2005.

[8] S. Ozdem, M. Akcam, A. Yilmaz, M. Gultekin, and R. Artan, "Biochemical markers of bone metabolism in children with Helicobacter pylori infection," Digestive Diseases and Sciences, vol. 52, no. 4, pp. 967-972, 2007.

[9] A. M. Kakehasi, C. M. C. Mendes, L. G. V. Coelho, L. P. Castro, and A. J. A. Barbosa, "The presence of Helicobacter pylori in postmenopausal women is not a factor to the decrease of bone mineral density," Arquivos de Gastroenterologia, vol. 44, no. 3, pp. 266-270, 2007.

[10] J. R. Warren and B. J. Marshall, "Unidentified curved bacilli on gastric epithelium in active chronic gastritis," The Lancet, vol. 1, no. 8336, pp. 1273-1275, 1983.

[11] B. J. Marshall, J. R. Warren, and G. J. Francis, "Rapid urease test in the management of campylobacter-pyloridis-associated gastritis," American Journal of Gastroenterology, vol. 82, no. 3, pp. 200-210, 1987. 
[12] World Health Organization, Assessment of Fracture Risk and Its Application to Screening for Postmenopausal Osteoporosis: Report of a WHO Study Group, WHO, Geneva, Switzerland, 1994.

[13] I. R. Garrett, B. G. M. Durie, and G. E. Nedwin, "Production of lymphotoxin, a bone-resorbing cytokine, by cultured human myeloma cells," The New England Journal of Medicine, vol. 317, no. 9, pp. 526-532, 1987.

[14] M. Akçam, "Helicobacter pylori and micronutrients," Indian Pediatrics, vol. 47, no. 2, pp. 119-126, 2010.

[15] K. L. Tucker, M. T. Hannan, N. Qiao et al., "Low plasma vitamin $B_{12}$ is associated with lower BMD: the Framingham Osteoporosis study," Journal of Bone and Mineral Research, vol. 20, no. 1, pp. 152-158, 2005.

[16] M. Herrmann, J. P. Schmidt, N. Umanskaya et al., "The role of hyperhomocysteinemia as well as folate, vitamin $\mathrm{B}_{6}$ and $\mathrm{B}_{12}$ deficiencies in osteoporosis-a systematic review," Clinical Chemistry and Laboratory Medicine, vol. 45, no. 12, pp. 16211632, 2007.

[17] L. E. Targownik, W. D. Leslie, K. S. Davison et al., "The relationship between proton pump inhibitor use and longitudinal change in bone mineral density: a population-based from the Canadian Multicentre Osteoporosis Study (CaMos)," American Journal of Gastroenterology, vol. 107, no. 9, pp. 1361-1369, 2012.

[18] Y. T. Lau and N. N. Ahmed, "Fracture risk and bone mineral density reduction associated with proton pump inhibitors," Pharmacotherapy, vol. 32, no. 1, pp. 67-79, 2012. 


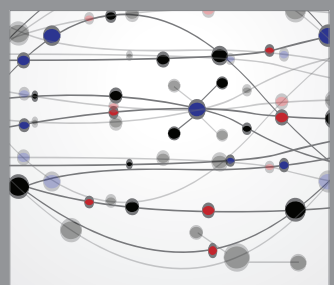

The Scientific World Journal
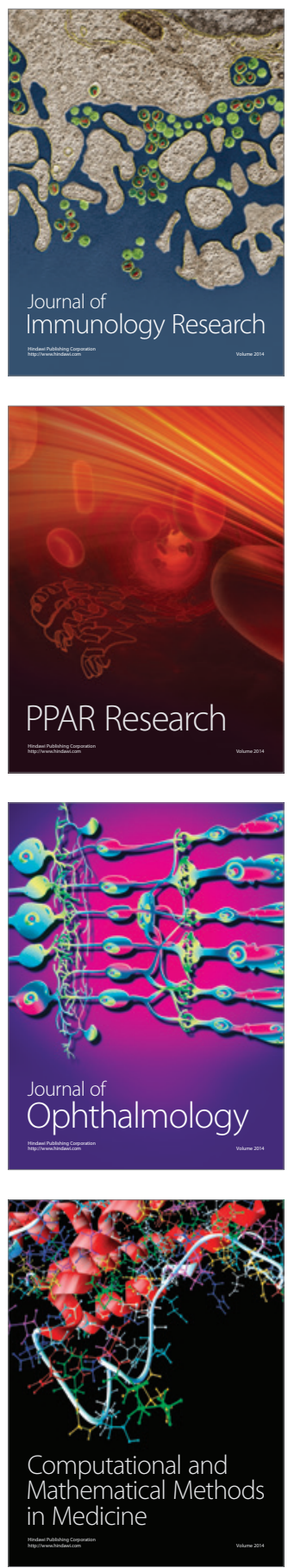

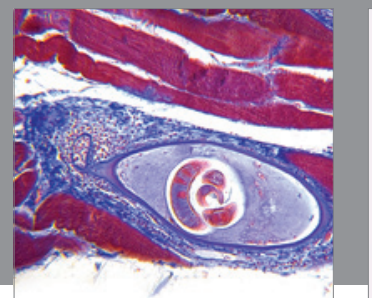

Gastroenterology

Research and Practice
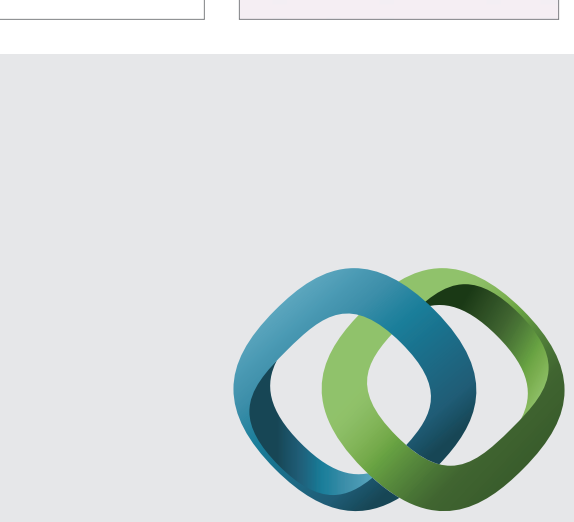

\section{Hindawi}

Submit your manuscripts at

http://www.hindawi.com
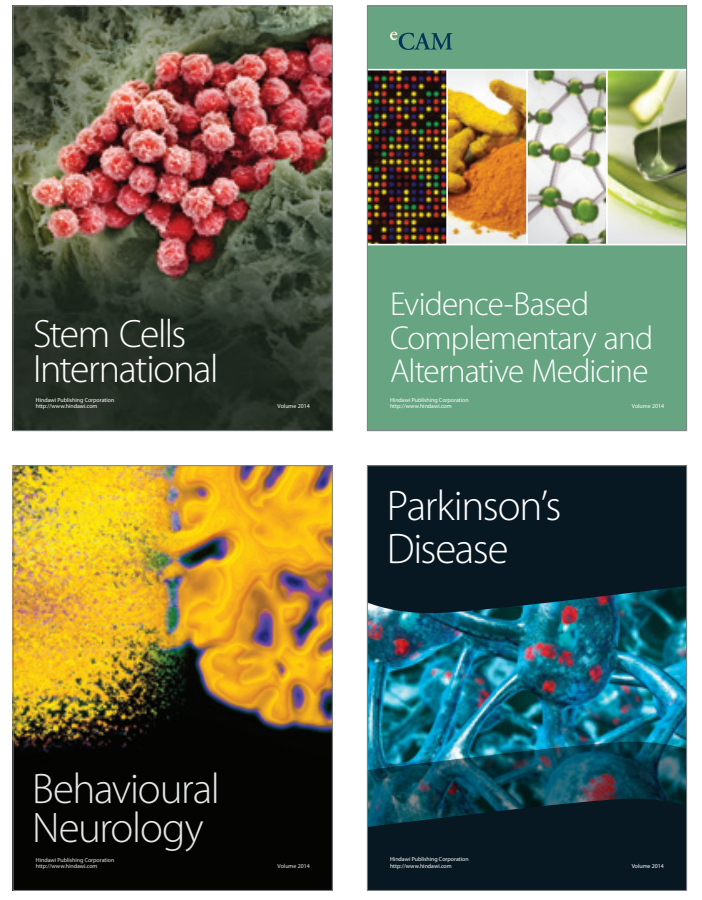


Disease Markers
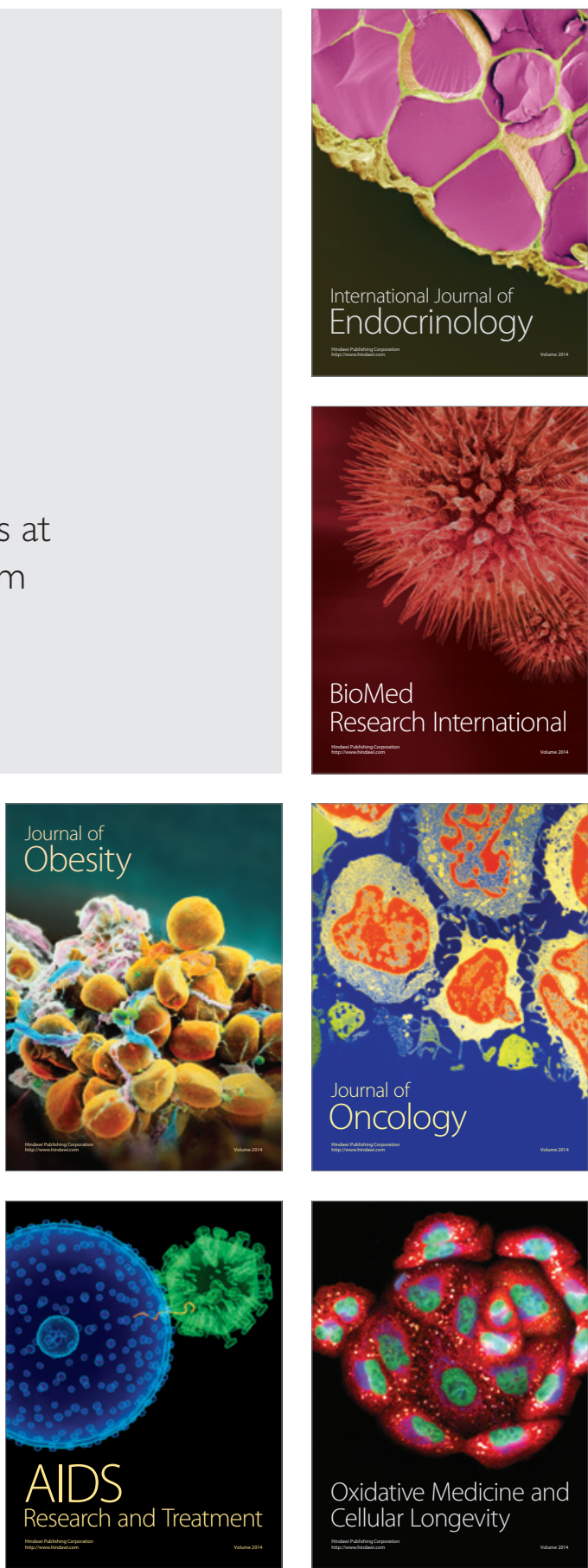